\title{
Protector Role of Intraocular Lenses under Artificial Light Conditions
}

\author{
Andrés Fernández-Vega Cueto ${ }^{a, b}$ Susana del Olmo-Aguado ${ }^{a, c}$ \\ Eva García-Pérez ${ }^{b}$ Ignacio Rodriguez-Uña ${ }^{b}$ Luis Fernández-Vega Cueto ${ }^{a, b}, c$

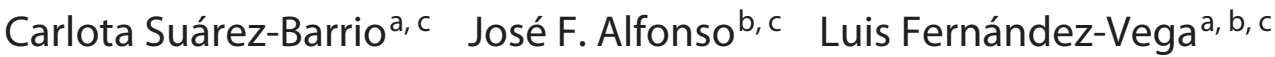 \\ Jesús Merayo-Lloves ${ }^{\mathrm{a}, \mathrm{c}}$ \\ aUniversity Institute Fernández Vega, Ophthalmologic Research Foundation, University of Oviedo, \\ Oviedo, Spain; 'bernández-Vega Ophthalmological Institute, Oviedo, Spain; ' $H$ Health Research Institute of \\ Asturias (ISPA), Oviedo, Spain
}

\section{Keywords}

Short wavelength blue light · Filters · Intraocular lenses ·

Protection

\begin{abstract}
Introduction: The aim of this work was to analyze, in an in vitro model, the possible protective effects of ultraviolet(UV-) or UV/blue-filtering intraocular lens (IOL) under lightemitting diode (LED) lighting conditions. Methods: Ten models of IOLs were evaluated. Light transmission spectrum was recorded from 300 to $800 \mathrm{~nm}$, in steps of $1 \mathrm{~nm}$. Photodamage in vitro model was induced in ARPE-19 cells by blue LED light (465-475 nm). Changes in cell viability and oxidative stress variables were studied to assess the protective effect of IOLs. Results: UV/blue-filtering IOLs models block blue light spectrum in different proportion and UV-filtering IOLs blocking wavelength below $400 \mathrm{~nm}$. However, in vitro study under blue LED light exposure does not show protective effects related with mitochondrial dysfunction and oxidative stress of UV/blue-filtering IOLs. Conclusions: The current in vitro study suggests that UV/blue filtering IOLs are
\end{abstract}

karger@karger.com www.karger.com/ore

Karger $\stackrel{\text { ' }}{=}$
(C) 2021 The Author(s)

Published by S. Karger AG, Basel

This is an Open Access article licensed under the Creative Commons Attribution-NonCommercial-4.0 International License (CC BY-NC) (http://www.karger.com/Services/OpenAccessLicense), applicable to the online version of the article only. Usage and distribution for commercial purposes requires written permission. not useful in terms of photoprotection in artificial light conditions. The results obtained indicate that it is needed to give attention to other IOL parameters besides the type of filter, as it seems they could have influence on the protective role.

(C) 2021 The Author(s).

Published by S. Karger AG, Basel

\section{Introduction}

The influence of artificial light systems, such as devices using light-emitting diodes (LEDs), which have radiation peaks in the blue spectrum, is of current interest to the scientific community. A number of authors have shown the harmful effect that this type of lighting could produce on the retina, inducing mitochondrial dysfunction and the activation of apoptosis and necrosis in retinal ganglion cells (RGCs), photoreceptors, and retinal pigment epithelium (RPE) [1-3].

The phototoxic action of light has been linked to retinal diseases such as age-related macular degeneration (AMD) [4]. There is evidence demonstrating the effects of light and its involvement in the progression of the dis- 
ease [5-7]. Short-wavelength visible light produces increased production of reactive oxygen species (ROS), which may result in the activation of a variety of cell death mechanisms [8-11].

Oxidative stress has also been directly related to glaucoma, in which it causes damage at three sites: the trabecular meshwork, the optic nerve head, and the retina [12-14]. Visible short-wavelength light can interact with the cellular components of RGCs, and this may be an added risk factor in patients with glaucoma, where the stress level of RGCs has been found to increase due to the effects of light $[1,15,16]$.

The crystalline lens has an important function as a short-wavelength filter. Aging produces a "yellowing" of the lens which acts to diffuse and reduce the amount of light hitting the retina [17-19]. However, this reduction in the quantity and/or quality of patients' vision as a consequence of the opacification of the lens means the lens needs to be removed surgically and replaced with an intraocular lens (IOL).

The first IOLs that were produced did not contain light filters: as such, patients who had their lens removed and replaced with a filterless IOL were exposed to higher levels of ultraviolet (UV) and visible light [20], to the detriment of the retina. Later, IOLs with a UV filter integrated into them were developed, thus reproducing the natural filtering capacity of the lens. Indeed, all IOLs on the market contain UV-radiation filters, and it is increasingly common that IOLs containing a yellow filter in order to reduce/block blue spectrum wavelengths are also developed. Scientific evidence demonstrating the impact of short-wavelength visible light on the retina has led to the development of IOLs which incorporate new filters for this type of radiation [21,22]. However, experimental studies regarding the utility of UV/blue-filtering IOLs are extremely reduced, and the clinical studies are contradictory. Some authors indicate that using UV/bluefiltering IOLs causes quality of vision impairment [23] or have not detected protective effects after implantation [24]. Conversely, other authors report that the use of UV/blue-filtering IOLs was useful in retinal protection [25]. Here, we show the first in vitro study analyzing a large number of IOL models in a controlled environment to elucidate the erratic effect of the use of UV/blue-filtering IOLs.

It should be noted that IOLs were developed in order to protect from solar radiation. However, during the last decades, population has increased the exposure to artificial light, which presents different spectrum than natural light.

Protector Role of Intraocular Lenses
Given the increasing dependency to artificial light, it becomes especially relevant to evaluate the protective effect of IOLs under these conditions, especially in the case of preexisting diseases, such as glaucoma or AMD, in which the survival of retinal cells is already compromised. The aim of this work was to analyze the protective behavior of ten commercial IOLs, containing either a UV filter or a UV/blue filter, in a retinal in vitro model.

\section{Materials and Methods}

IOLs

Ten different models of either UV-filtering or UV/blue-filtering IOLs were analyzed (Table 1). The dioptric range employed was from 20.0 to $20.5 \mathrm{D}$.

\section{Measurement of Transmittance}

Light transmission was measured, every nm, between 300 and $800 \mathrm{~nm}$ for the IOLs selected for the study. An experimental setup was designed for this purpose whose light source consisted of two lamps: one, a xenon model - L2274 Hamamatsu (source C8849; Hamamatsu) - for measurements in the range $350-450 \mathrm{~nm}$, and the other, an incandescent model - LAES 12 V, $50 \mathrm{~W}$ (source 6286; $\mathrm{HP}$ ) - for measurements between 450 and $900 \mathrm{~nm}$. In addition, the setup incorporated a chamber, into which each IOL was placed. This was formed of two metal end pieces joined by two pieces of Teflon with frosted glass in the exit window and in contact with the monochromator MonoSpec 18; F/3.8 (Thermo Jarrell-Ash filial de; Thermo Fisher Scientific, Waltham, MA, USA), along with a diaphragm and housing for the IOL. Once the chamber was screwed together, it was filled with $0.9 \%$ saline solution to simulate the intraocular environment. As well as the monochromator mentioned above, which dispersed the light from the source lamps, the equipment also incorporated an optical multichannel analyzer with 509 channels which detected the amount of light that passed through the IOL, and a computer which recorded the signal.

\section{Cell Cultures}

The cell line ARPE-19 (CRL-2302 ${ }^{\mathrm{TM}}$, ATCC $^{\circledR}$; Manassas, VA, USA), which is derived from human RPE was used. For the purpose of the experiment, the ARPE-19 cells were seeded at a concentration of $1 \times 10^{4}$ cells/mL in DMEM:F12 medium supplemented with $10 \%$ fetal bovine serum (FBS) and $1 \%$ penicillin/streptomycin antibiotics in an atmosphere of $5 \% \mathrm{CO}_{2}$ at $37^{\circ} \mathrm{C}$ for a period of $24 \mathrm{~h}$ for the culture to stabilize. Prior to light exposure, the medium was changed to one which was almost the same except that the concentration of FBS was lowered to $1 \%$.

Light Exposure

ARPE-19 monolayers were maintained in dark conditions (control group) or exposed to blue (465-475 nm, $400 \mathrm{lux}, 17 \mathrm{~W} /$ $\mathrm{m}^{2}, 36 \mathrm{~h}$ ) LEDs lights (Electro DH, SL, Barcelona, Spain) with or without IOLs.

\section{Cell Viability}

Assessment of cell viability was carried out using WST-1 (Roche Diagnostics, S.L., Barcelona, Spain) that consist in a colo- 
Table 1. Summary of IOLs characteristics employed in the study

\begin{tabular}{llll}
\hline Type of filter & Model & Optics & Material \\
\hline UV filter & Tecnis Aspheric IOL PCB800 Johnson \& Johnson & Monofocal & Hydrophobic acrylic \\
& CT Lucia 611P Zeiss & Monofocal & Hydrophobic acrylic \\
& AT Lisa 809M Zeiss & Bifocal & Hydrophilic acrylate with hydrophobic surface \\
& AT Lisa Tri 839 MP Zeiss & Trifocal & Hydrophilic acrylate with hydrophobic surface \\
& Tecnis Symfony ZXR00 Johnson \& Johnson & EDOF & Hydrophobic acrylic \\
\hline UV/blue filter & Acrysoft IQ SN60WF Alcon & Monofocal & Hydrophobic acrylic \\
& Acrysoft Restor SV25T0 Alcon & Bifocal & Hydrophobic acrylic \\
& Acrysoft IQ Restor SN6AD1 Alcon & Bifocal & Hydrophobic acrylic \\
& Acrysoft IQ Panoptix TFNT00 Alcon & Trifocal & Hydrophobic acrylic \\
& Physiol Fine Vision PODF & Trifocal & Acrylic copolymer with hydrophobic surface \\
\hline
\end{tabular}

rimetric assay that quantifies mitochondrial dehydrogenase activity. Briefly, ARPE-19 cells were seeded, grown, and then, incubated for $36 \mathrm{~h}$ in dark or under light conditions, as described above. After light exposure, cells were allowed to react with $10 \mu \mathrm{L} /$ well WST-1 reagent for $4 \mathrm{~h}$. Thereafter, the optical density of formazan dye formed was recorded at $450 \mathrm{~nm}$ using an automated microplate reader (PerkinElmer, Waltham, MA, USA).

\section{Immunocytochemistry}

Cells were fixed in $4 \%$ paraformaldehyde for $15 \mathrm{~min}$ at room temperature. Following incubation, the samples were twice washed for $10 \mathrm{~min}$ with phosphate-buffered saline (PBS). After washing and fixing the cells were permeabilized with $0.1 \%$ Triton-X100/ PBS for 10 min and then washed once again in PBS and blocked in $10 \%$ goat serum for $1 \mathrm{~h}$. At the end of this time, the samples were incubated overnight at $4^{\circ} \mathrm{C}$ with one of the following primary antibodies: anti-heme oxygenase-1 (HO-1) (Enzo Life Sciences, Farmingdale, NY, USA) 1:50, anti-nuclear factor kappa-lightchain-enhancer of activated B cells (NFkB) (Santa Cruz Biotechnology, Dallas, TX, USA) 1:50, or anti-ZO-1 (Thermo Fisher, Rockford, IL, USA) 1:100. The next day the samples were again washed with PBS and incubated with a secondary antibody labeled with fluorophore - either Alexa Fluor 488 or Alexa Fluor 594 (Thermo Fisher Scientific) - at a concentration of 1:300 for $2 \mathrm{~h}$ at room temperature. The final step was to add DAPI $(0.2 \mu \mathrm{g} / \mathrm{mL})$ as a nuclear stain and store the cells in PBS. Visualization of samples was carried out using a Leica DMI6000B inverted fluorescence microscope (Leica Microsystems GmbH, Wetzlar, Germany).

\section{Mitochondrial Membrane Potential}

Changes in mitochondrial membrane potential were detected using the commercial kit JC-1 (Sigma-Aldrich, St. Louis, MO, USA). JC-1 accumulates in mitochondria appearing as red fluorescence in healthy organelles and green fluorescence when the membrane is depolarized. Briefly, JC-1 dye solution $(2 \mu \mathrm{g} / \mathrm{mL})$, freshly prepared in buffer solution, was added to cultures for $20 \mathrm{~min}$ at $37^{\circ} \mathrm{C}$ and $5 \% \mathrm{CO}_{2}$. Next, the excess dye was removed, and the sample was washed with fresh culture medium after which it was visualized under the microscope Leica DMI6000B. Three randomized areas of $448.92 \times 335.40$ microns of each one were photographed to produce a quantitative analysis of JC-aggregates/monomer ratio with ImageJ software (ImageJ 1.46r; NIH, Bethesda, MD, USA).
Detection of ROS

To detect ROS the nonfluorescent compound dihydroethidium (DHE) (Thermo Fisher Scientific) was used. DHE has the ability to passively enter live cells, where it is oxidized by the anion superoxide. Following the period of light exposure, the cell medium was changed and DHE was added at a concentration of $40 \mu \mathrm{M}$. After 30 min of incubation at $37^{\circ} \mathrm{C}$ and $5 \% \mathrm{CO}_{2}$, the medium was removed and the cells washed in fresh medium after which they were immediately analyzed with fluorescent microscopy using a Leica DMI6000B inverted fluorescence microscope. Quantitative analysis of ROS production was determined using ImageJ software. Five randomized areas of $448.92 \times 335.40$ microns of each sample were photographed and analyzed.

\section{Statistical Analysis}

The results data are shown as the mean \pm standard error of the mean and were analyzed with the statistics programme Prism 6 (GraphPad Software, La Jolla, CA, USA), and one-way ANOVA, with Fisher's LSD test, was performed. In the case of nonparametric data, Kruskal-Wallis test was used. The minimal difference was set at $p<0.05$.

\section{Results}

\section{Light Transmission in IOLs}

The transmittance measurements revealed differences between IOLs in their patterns of filtering UV and blue spectra wavelengths (Fig. 1). Since the resulting curve for Alcon Acrysof IQ IOL was in accord with previously published data $[26,27]$, the validity of our findings can be assumed.

In terms of the IOL models with UV filters, Tecnis PCB00 and Tecnis ZXR00 blocked wavelengths below $370 \mathrm{~nm}$, while AT Lisa $839 \mathrm{MP}$ and CT Lucia blocked those below $360 \mathrm{~nm}$ and AT Lisa 809 achieved 100\% blocking of wavelengths below $350 \mathrm{~nm}$. All four models showed a precipitous increase in the transmission of light 
Fig. 1. Differences in the pattern of light transmission between the different IOLs. All models studied here except TFNT00, SN6AD1, SN60WF, and SV25T0 allowed the passage of wavelengths below $400 \mathrm{~nm}$. The UV-filtering IOLs all showed similar transmission patterns with a sharp change of transmittance around $390 \mathrm{~nm}$. In contrast, two different patterns were observed for the UV/blue-filtering IOL models. The first, that of the Pod F model, shows rapidly increasing transmission up to $400 \mathrm{~nm}$ (as for the UV-filtering lenses) before plateauing until $450 \mathrm{~nm}$, and then increasing more gradually. The second pattern corresponds to the other UV/blue-filtering IOLs (TFNT00, SN6AD1, SN60WF, and SV25T0), where transmission increased steadily between $390 \mathrm{~nm}$ and $490 \mathrm{~nm}$.

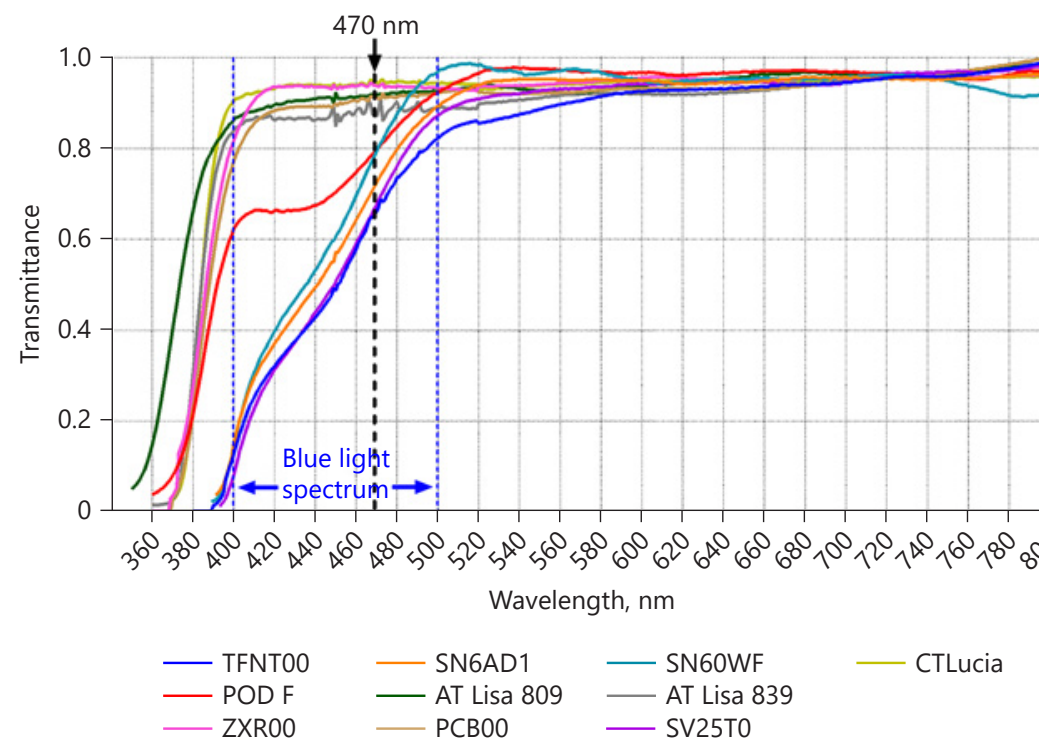

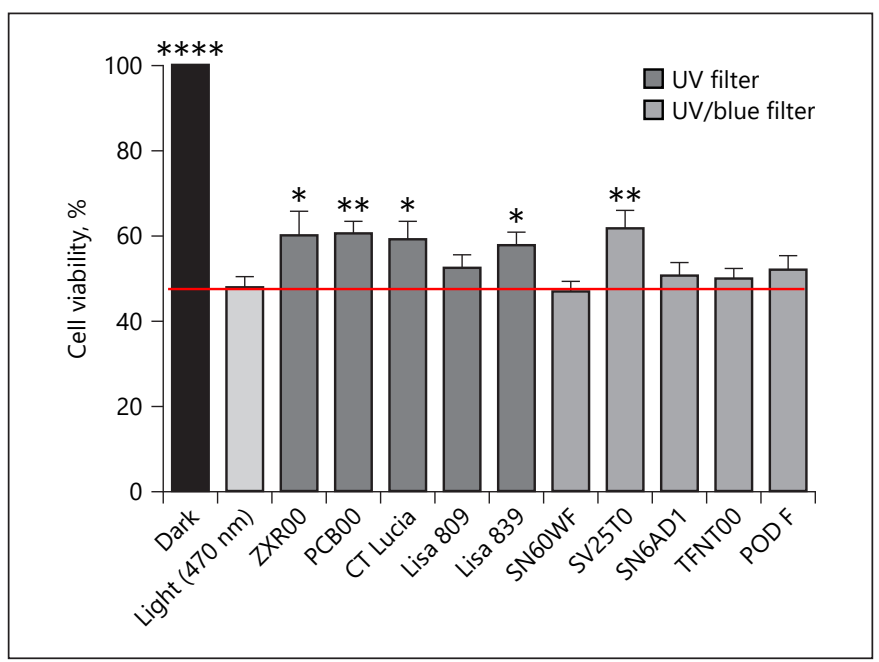

Fig. 2. Viability with the use of IOLs. ARPE-19 cells were exposed to darkness or LED blue light (470 nm, 400 lux, $17 \mathrm{~W} / \mathrm{m}^{2}, 36 \mathrm{~h}$ ), either with or without the use of an IOL. At the end of the exposure period, the number of viable cells was quantified with WST-1. Oneway ANOVA was performed, Fisher's LSD test showed significant differences between the IOLs used under blue light respect to light group without IOLs, ${ }^{*} p<0.05,{ }^{* *} p<0.01,{ }^{* * *} p<0.0005$, ${ }^{* * * *} p<$ 0.0001 . The results are shown as mean \pm SEM, where $N=9$.

up to $390-400 \mathrm{~nm}$ where transmission levels were $80 \%$. Above $400-525 \mathrm{~nm}$, all the UV-filtering IOLs studied here reached a transmittance of over $90 \%$, which was maintained up to $800 \mathrm{~nm}$. Of particular interest are the
Table 2. Percentage transmittance to $380 \mathrm{~nm}, 400 \mathrm{~nm}$, and $470 \mathrm{~nm}$ of IOLs analyzed

\begin{tabular}{llll}
\hline Model & $\begin{array}{l}\text { \% Transmittance } \\
380 \mathrm{~nm}\end{array}$ & $\begin{array}{l}\text { \% Transmittance } \\
400 \mathrm{~nm}\end{array}$ & $\begin{array}{l}\text { \% Transmittance } \\
470 \mathrm{~nm}\end{array}$ \\
\hline PCB00 & 21.4 & 77.2 & 91.2 \\
CTLucia & 27.4 & 90.5 & 94.5 \\
AT Lisa 809 & 66.1 & 86.3 & 91.8 \\
AT Lisa 839 & 30.4 & 84.0 & 87.1 \\
ZXR00 & 27.6 & 81.8 & 93.8 \\
SN60WF & 0.0 & 14.7 & 79.4 \\
SV25T0 & 0.0 & 8.2 & 67.8 \\
SN6AD1 & 0.0 & 15.3 & 72.4 \\
TFNT00 & 0.0 & 12.8 & 66.1 \\
PODF & 21.4 & 62.3 & 79.6 \\
\hline
\end{tabular}

data for the wavelength $470 \mathrm{~nm}$ (Table 2), which is that used for the in vitro phototoxicity experiment and also the maximum emission peak of devices using common white LEDs. The Tecnis PCB00 and Tecnis ZXR00 had transmittances of $91.2 \%$ and $93.8 \%$, respectively, at 470 nm, while the AT Lisa 839, CT Lucia, and AT Lisa 809 had values of between $87.1 \%$ and $94.5 \%$. In terms of the UV/ blue-filtering IOLs examined in this work, the Fine Vision POD F blocked wavelengths below $360 \mathrm{~nm}$, and its transmittance increased by $60 \%$ at $400 \mathrm{~nm}$. Transmittance between 400 and $450 \mathrm{~nm}$ was quite stable, only varying by $10 \%$, but above $450 \mathrm{~nm}$ there was a sharp increase in transmission with the passage of $90 \%$ of light at 


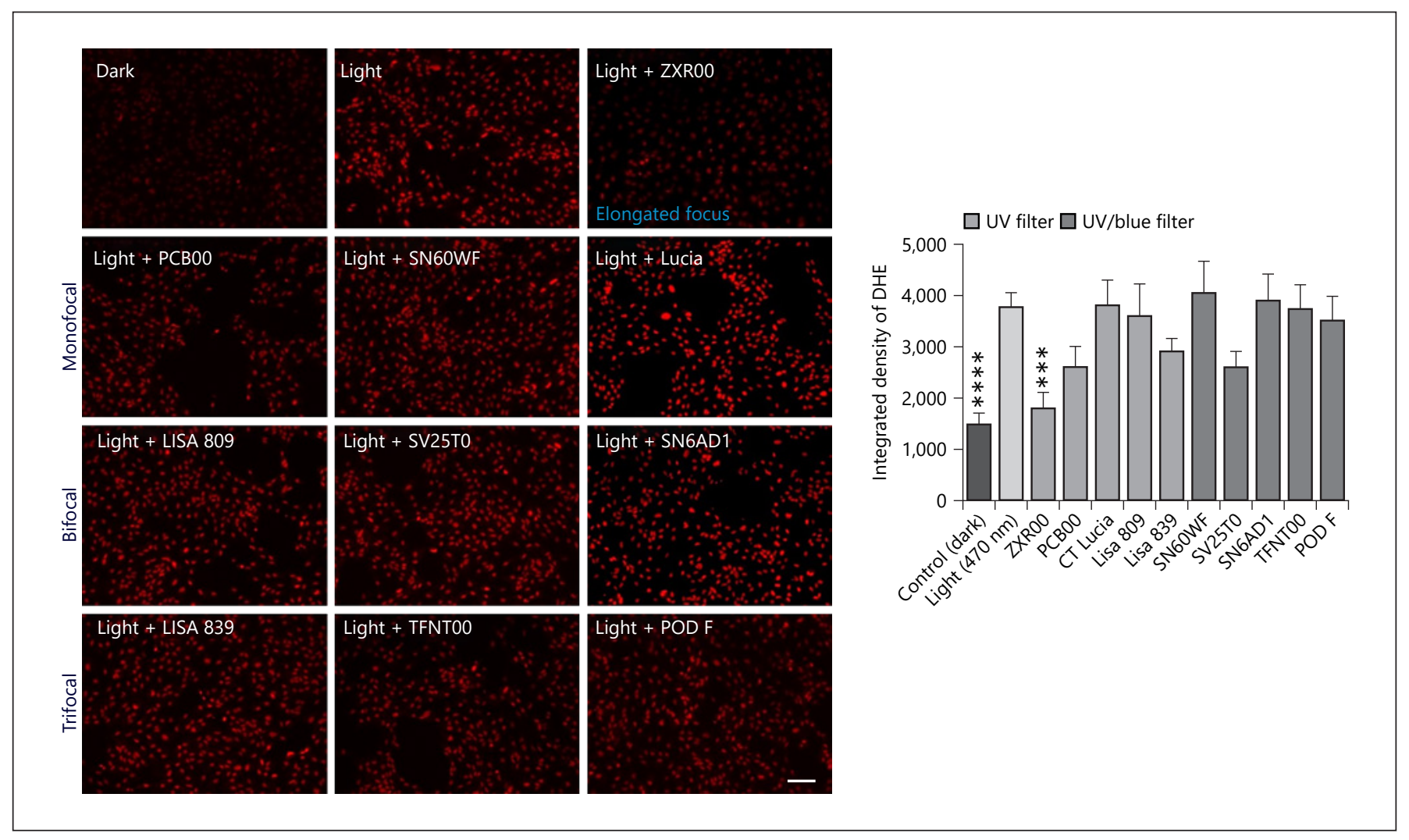

Fig. 3. Interaction between light, IOL, and ROS. ARPE-19 cells exposed to light did not present a reduction in ROS levels as a result of the use of IOLs. Only the model Tecnis Symfony ZXR00 showed a significant reduction compared to light. Scale $50 \mu \mathrm{m}$. The diagram represents the integrated density of DHE fluorescence. An ANOVA study confirms the data observed. Kruskal-Wallis test showed ${ }^{* * *} p<0.0005$ (to ZXR00), ${ }^{* * * *} p<0.0001$ (to Dark) compared to light. Results are shown as mean \pm SEM, where $N=4$.

$492 \mathrm{~nm}$. The models (PanOptix, ReStor SV25T0, and SN6AD1), and Acrysof SN60WF blocked all wavelengths up to $390 \mathrm{~nm}$, after which there was a gradual increase in transmittance up to $490 \mathrm{~nm}$, where it leveled off and all these models demonstrated values of over $80 \%$. For the PanOptix model, there was approximately $5 \%$ less transmission between $390 \mathrm{~nm}$ and $490 \mathrm{~nm}$ than for the other two models, which are from the same manufacturer. At $470 \mathrm{~nm}$ transmittance for the PanOptix, Restor (SV25T0 and SN6AD1) and Acrysof IOLs was, respectively, 66.1\%, $67.8 \%, 72.4 \%$, and $79.4 \%$ and for the Fine Vision POD F it was $79.6 \%$. Between $485 \mathrm{~nm}$ and $565 \mathrm{~nm}$, the UV/bluefiltering IOLs reached transmittances of over $90 \%$, and this was maintained up to $800 \mathrm{~nm}$.

\section{Effect of IOL Use in the in vitro Model of RPE}

Blue light negatively affected RPE cells. The use of IOLs produced a variety of results (Fig. 2) with the models Tecnis ZXR00, Tecnis PCB00, CT Lucia, Restor
SV25T0, and Lisa 839 IOLs all demonstrating significant effects against damage. Of these, Restor SV25T0 was UV/ blue-filtering.

Exposure to light of $470 \mathrm{~nm}$ produced an increase in ROS in ARPE-19 cells that were not detected in cells kept in dark. The use of IOLs did not alter the effect of this light, except in the case of the Tecnis ZXR00 model, where a reduction in ROS was noted compared to when they were exposed directly to light of this wavelength (Fig. 3).

The mitochondrial membrane potential was found to be affected by exposure to phototoxic stimuli. The use of IOLs during such light exposure resulted in a modest reduction in the depolarization of the mitochondrial membrane (Fig. 4a), although this effect varied between the different IOL models. Although JC-1 is not considered a mitochondrial marker, red dye of polarized mitochondrial membranes is distributed along mitochondrial structure. Elongated structures were observed in control cells. However, light exposure reduced to dots through 

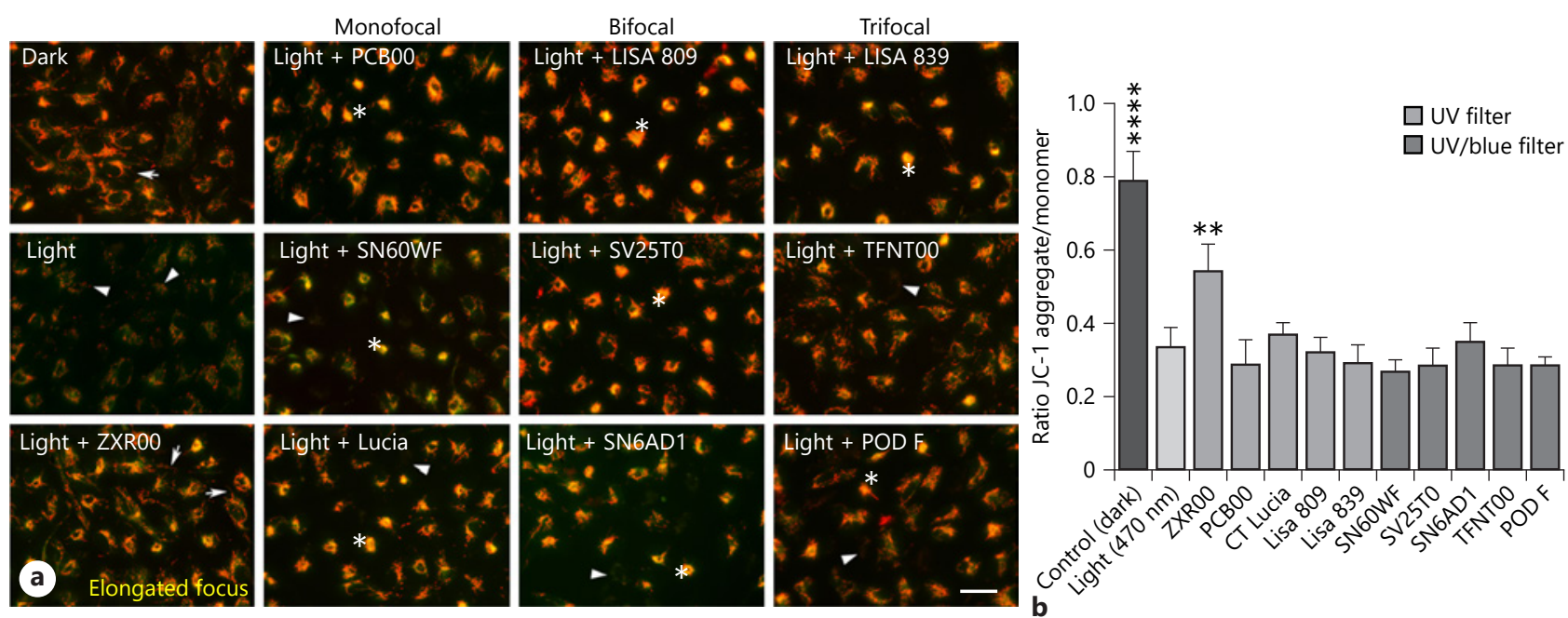

Fig. 4. a, b Conservation of mitochondrial membrane potential with the use of IOLs. The analysis of the state of mitochondrial membrane potential using the dye JC-1 showed the harmful effect of light of $470 \mathrm{~nm}$ on ARPE-19 cells. The use of IOLs reduced, in a general way, alterations in mitochondrial membrane potential. Scale $50 \mu \mathrm{m}$. Graphic represents the ratio of JC-aggregates/monomer. One-way ANOVA was performed. Fisher's LSD test showed significant differences to ZXR00 and dark compared to light samples $\left({ }^{* *} p<0.01,{ }^{* * * *} p<0.0001\right)$. Results are shown as mean \pm SEM, where $N=4$.
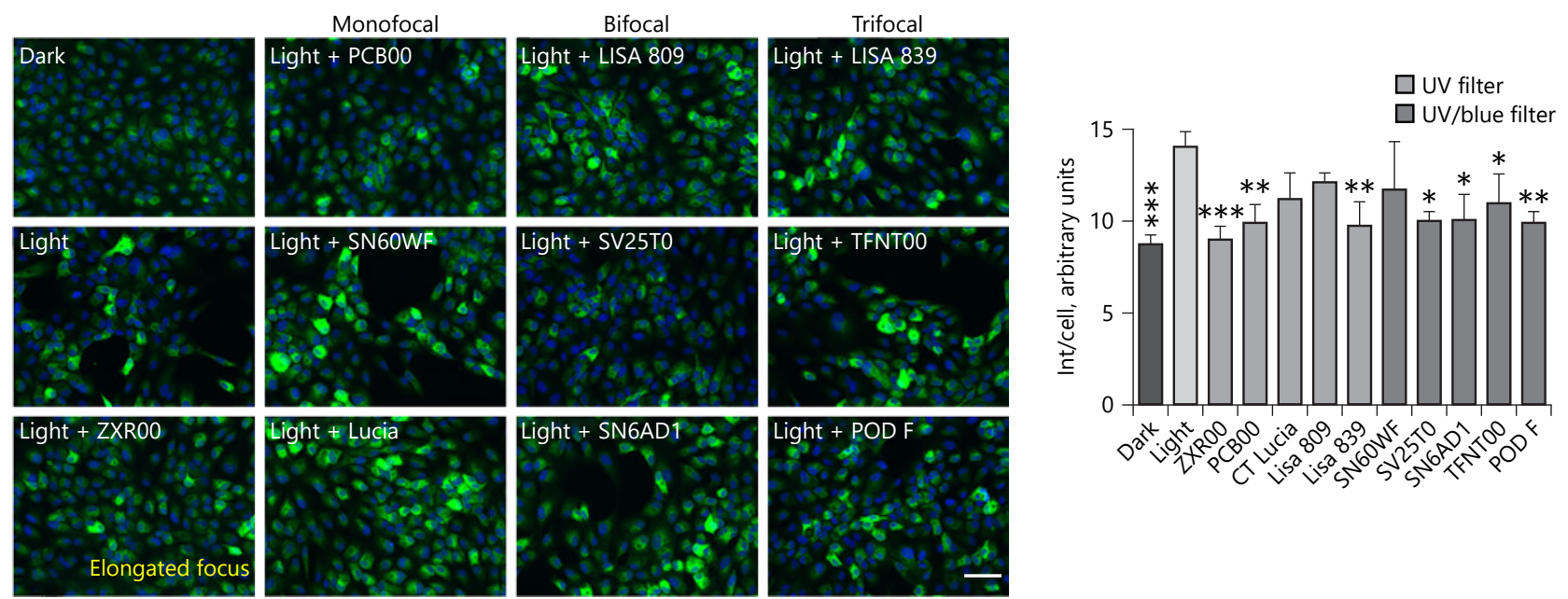

Fig. 5. Modification of the expression of proteins related to oxidative stress damage. HO-1 (green) immunofluorescence in ARPE19 cells in the dark (control) or after $36 \mathrm{~h}$ of exposure to light at $470 \mathrm{~nm}$ either with or without the use of an IOL. The light resulted in a variable increase in the expression of HO-1. In certain cases, the use of an IOL reduced expression, but in no instance did expression fall to the levels of control cells. Nuclei were stained with
DAPI. Scale $50 \mu \mathrm{m}$. Quantitative analysis of HO-1 expression was determined using ImageJ software. Graphic represents mean of intensity per cell. One-way ANOVA was performed. Fisher's LSD test showed significant differences to control, ZXR00, PCB00, SV25T, SN6AD1, Lisa 839, TFNT00, and POD F compared to light $\left({ }^{*} p<0.05,{ }^{* *} p<0.01,{ }^{* * *} p<0,0005\right)$. Results are shown as mean \pm SEM, where $N=4$. 


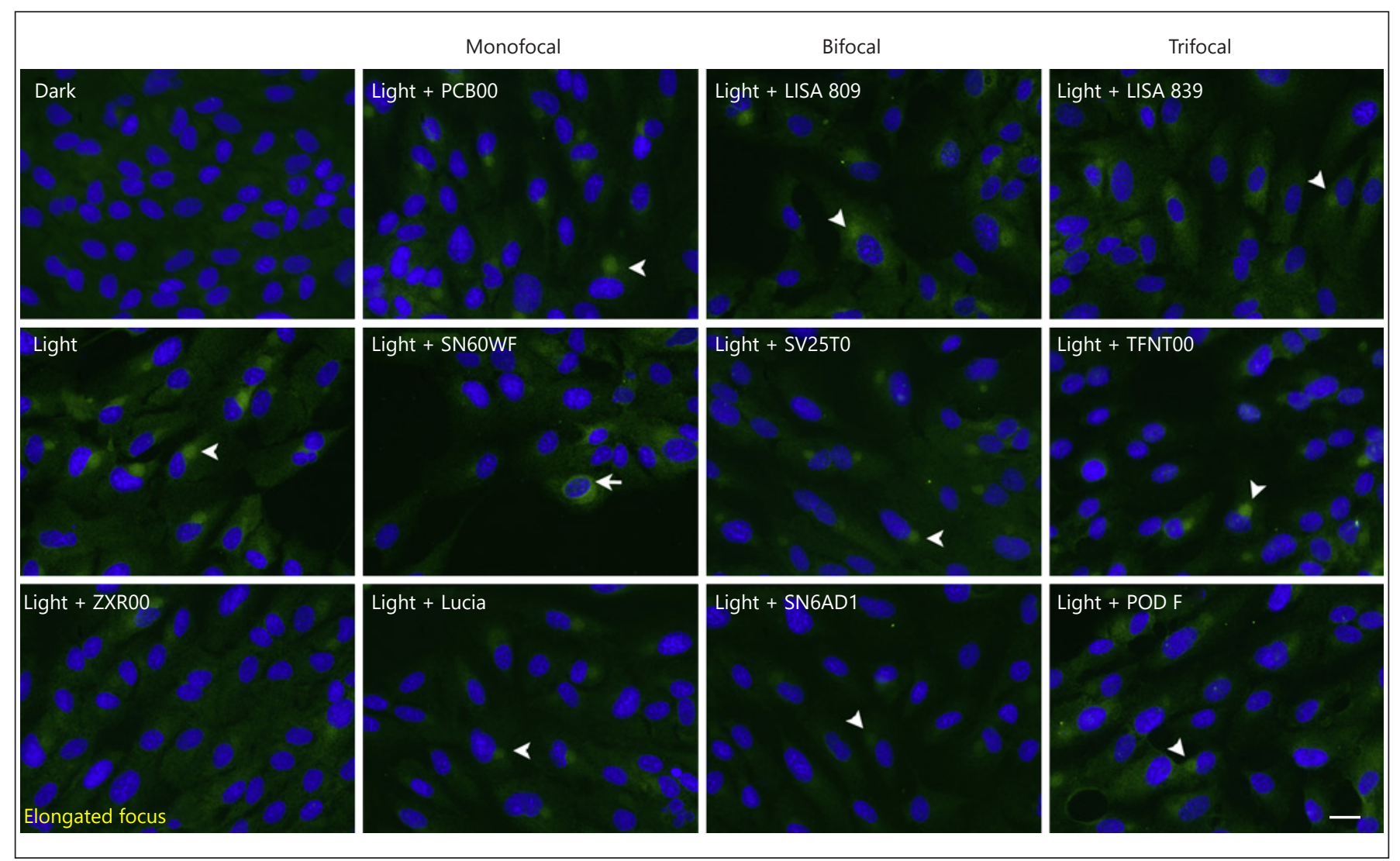

Fig. 6. Changes in expression of NFkB. Blue light produced changes in the expression of NFkB (green) with respect to ARPE-1 cells kept in the dark. NFkB was located in the cytoplasm in dark samples. After light exposure, NFkB was principally observed close to the nucleus (blue, DAPI) (arrow) and or into the nucleus (head arrow). The use of IOL slightly reduced NFkB expression for all the models studied here, although the strength of the effect differed between models. Scale $50 \mu \mathrm{m}$.

the distribution of polarized membranes. The use of IOLs reduced the loss of mitochondrial potential but the localization of the dye suggesting a rounding of cells prior detachment and death. Just in the case of Tecnis ZXR00, the cells showed levels and localization of dye similar to control cells. Quantitative data of JC-1 ratio showed a mitochondrial alteration in almost all of groups exposed to light and IOLs (Fig. 4b). Just the ZXR00 model reduced the impact of the light on the cells.

Interaction with the light produced molecular changes in ARPE-19 cells. Direct exposure increased expression of HO-1. None of the IOLs tested in this work reduced its expression to the levels detected in cells kept in darkness (Fig. 5), although the proportion of reactive cells was reduced in the case of the models Tecnis ZXR00, Tecnis PCB00c, and Restor SV25T0.

Changes were also detected in the NFkB (Fig. 6). NFkB translocates to the nucleus when cell damage occurs. We detected, at time of study, that light produces perinuclear accumulation of NFkB. Blue spectrum of light produced an alteration in the expression and distribution of NFkB, which was counteracted slightly by the use of IOLs, although the degree of reduction depended on the model employed. The use of Tecnis ZXR00 IOL reduced considerably to control levels the expression and localization of NFkB.

Visible short-wavelength light applied directly to the RPE cells produced a loss of cells which negatively impacted on tight junctions (Fig. 7). ZO-1 expression into the nucleus of ARPE-19 cell line has been detected. Light exposure produced loss of immunoreactivity in the intercellular junctions and increased the nuclear expression. The use of IOLs reduced the loss of this type of intercellular junction but not the nuclear expression. In the case of the ZXR00, IOL appears to preserve the tight junctions with an appropriate distribution. 


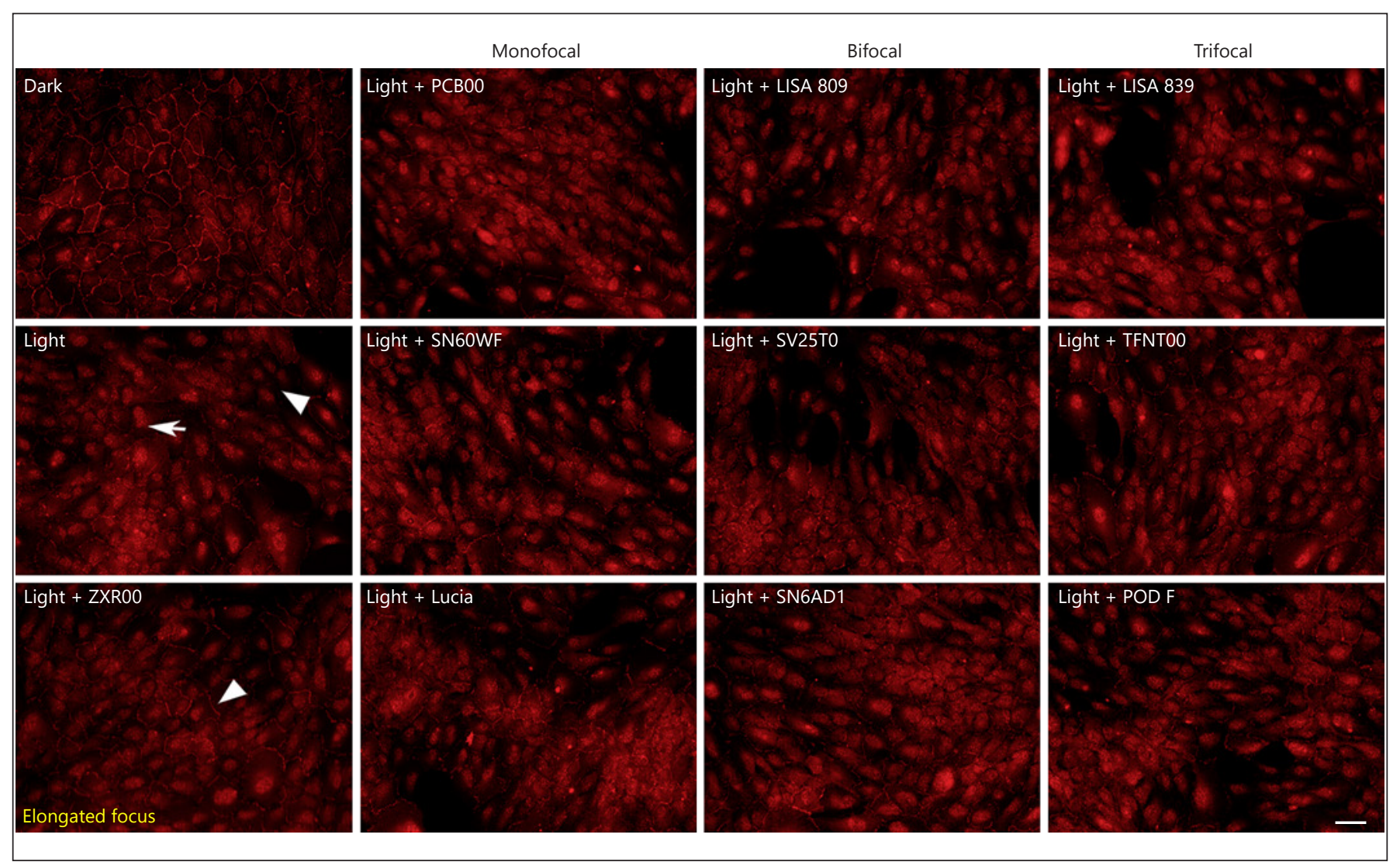

Fig. 7. Maintenance of cell structure with the use of IOLs. ZO-1 (red) immunofluorescence for the tight junctions of ARPE-1 cells. Light exposure (470 nm, $\left.400 \mathrm{lux}, 17 \mathrm{~W} / \mathrm{m}^{2}\right)$ produced a loss of ZO-1. The degree of integrity of the tight junctions depended on the IOL used. Scale $50 \mu \mathrm{m}$.

\section{Discussion}

Light interacts with ocular structures and is responsible for different biological responses depending on its range and target. Blue light (between 400 and $500 \mathrm{~nm}$ ) that impinges the retina is absorbed by various cellular components [10,16, 28,29]. Melanopsin, which is found in photosensitive ganglion cells [30-32], plays a fundamental role in the regulation of circadian rhythms and has a maximum excitation peak at $480 \mathrm{~nm}$ [33-35]. Other targets of blue light include mitochondria, where there are numerous components that absorb short wavelength light. Proteins such as porphyrins, which are found in the internal membrane of mitochondria, and the enzyme complexes of the mitochondrial electron transport chain, absorb wavelengths between 400 and $410 \mathrm{~nm}$. Other essential components of mitochondrial enzyme systems are flavins that, as with cytochrome P450 and the cytochrome oxidases, absorb wavelengths of $420-450 \mathrm{~nm}$. This interaction could result in the formation of ROS and lead to oxidative stress and cellular damage $[16,36,37]$.

The harmful effects of short wavelength light in the visible spectrum on the retina have been shown in various in vitro and in vivo models at the level of photoreceptors, RGCs and the RPE $[1,2,38-40]$. The phototoxic action of blue light alters both the formation and structure of the tight junctions of RPE cells, which are essential for retinal integrity and homeostasis. At the molecular level, its action on cell damage mediators is well known, for example, in the induction of HO-1 expression $[1,41]$, a cytoprotective protein which is activated under conditions of cell damage or stress and plays an important role in homeostasis and the response to oxidative stress [42]. Other significant markers, involved in various cellular processes, are also activated in response to phototoxic stimuli. For example, NFkB is involved in the regulation of the proliferation and expression of genes related to inflammation, 
immunity, oxidative metabolism, senescence, and programmed cell death $[43,44]$.

The crystalline lens has an important role as a short wavelength light filter. However, the interaction between this kind of light [45], as well as the aging process [23], produces the opacification of the lens, making necessary to remove it and to replace it by an IOL.

As IOLs have developed, a number of features which offer protection against the effects of light radiation on the retina of patients have been incorporated. The use of UV/ blue-filtering IOLs, while now widespread, has not been without controversy. The results of various studies of the use of blue-filtering IOLs suggest that they do not provide protection to the retina, and in addition, they have certain disadvantages in terms of their affecting the quality of vision, especially in mesopic and/or scotopic conditions [46]. In addition, they have been linked with changes to circadian rhythms [26, 47] and depression [25]. However, other authors have demonstrated the protective capacity of blue-filtering IOLs. Pipis and collaborators [24] observed significant differences using blue-filtering lenses which slowed the progress of geographic atrophy in AMD patients following cataract surgery. A clinical study recently published [48] showed no differences, in terms of neuroprotection, between the use of UV- or UV/blue-filtering IOLs in a cohort of more than 11,000 patients.

Given our results, in which UV/blue filter IOLs do not provide an expected protective effect to cells against blue light, being these IOLs those that show the highest percentage of blocking $470 \mathrm{~nm}$ light, a possible point of discussion of the study is the role of the light transmitted distribution in multifocal IOLs. The viability results obtained for the trifocal IOLs are consistent with the energy of the transmitted light set, with a higher survival observed in the IOL that has a lower distribution in the main focus, the Lisa 839. Thus, it could be thought that the protective effect of the IOL under LED lighting conditions is given by the percentage of light distributed rather than by the type of filter present and, if so, the use of UV/blue-filtering IOL available not only would not provide benefits for photoprotection effects but also could involve alterations in the quality of vision [46]. However, that does not explain the results obtained with the monofocal and bifocal IOLs.

The set of in vitro results in the current work suggests that the filters involved do not play an important role in the potential protective effect of IOLs, and could instead depend on other parameters such as the optics and the materials. The optic may be an important factor since the study has involved both monofocal and multifocal (bifocal, trifocal, and extended depth of focus) IOLs, and in fact, the extended depth of focus lens (Tecnis ZXR00) was the one which provided the most consistent results. The posterior optic of the Tecnis ZXR00 IOL has an achromatic diffractive surface designed to correct chromatic aberration.

The material is another important parameter to consider. Although the IOLs tested in this study are all composed of one of the two principal materials used for implants - either hydrophobic acrylic or hydrophilic acrylic with a hydrophobic surface - there are differences between models in terms of their hydration level and the polymer used in their manufacturing process. These differences could impact on the light transmission capacities of the different lenses. The idea that the platform of IOL could be important is consistent with the results obtained. PCB00 slightly reduces (although not statistically significant in all parameters analyzed) the negative effect of light, not being the case for SN60WF. Those IOLs differ in the filter and platform. The Tecnis material has not been associated with glistening, whereas Acrysof showed this type of material degradation that now has been solved.

It is also of interest to note that the results of the transmittance measurements indicate that, although all the IOL models these days incorporate a UV filter, the majority of the IOLs tested here allowed the passage of shortwave radiation, with values ranging from $20 \%$ at $380 \mathrm{~nm}$ to $45 \%$ at $390 \mathrm{~nm}$. The exceptions to this were the SN60WF, TFNT00, SV25T0, and SN6AD1 IOLs. Wavelengths in the UV range are more reactive, and consequently more harmful than longer wavelengths. For this reason, although some IOL models which have only a UV filter have demonstrated greater protection under artificial lighting conditions in vitro, this effect may be diminished in natural light conditions, where solar radiation has a considerable UV component.

The current in vitro study suggests that UV/blue-filtering IOLs are not useful in terms of photoprotection in artificial light conditions. Furthermore, clinical evidence supporting the protective role of UV/blue-filtering IOLs is limited and highlights the need for more in depth study of this type of ophthalmic aid. This would help ophthalmologists to select a more appropriate IOL for the patient's requirements.

\section{Acknowledgment}

The authors are grateful to Santiago Mar for his technical support in the measurement of transmittance. 


\section{Statement of Ethics}

The immortalized cell line used in this study were purchased from ATCC $^{\circledR}\left(\mathrm{CRL}-2302^{\mathrm{TM}}\right)$. Ethical approval for the use of these cells is not required in accordance with local/national guidelines.

\section{Conflict of Interest Statement}

The authors have no conflicts of interest to declare.

\section{Funding Sources}

Part of this work was supported by the Project RTC-2016-4964: a project sponsored by the Spanish Ministry of Economy and Competitiveness, the European Union (through FEDER funds), and the Spanish State Research Agency. Another part of the research was funded by IDEPA, the European Union (through FEDER funds), and the PCTI, through the project IDE/2017/000653.

\section{Author Contributions}

Conceptualization: S.O.-A., A.F.-V.C., J.F.A., J.-M.L., L.F.-V.C. Methodology: A.F.-V.C., S.O.-A., E.G.-P., C.S.-B., L.F.-V.C. Investigation: A.F.-V.C., S.O.-A., E.G.-P., C.S.-B. Writing- original draft preparation: A.F.-V.C., S.O.-A., I.R.-U., L.F.-V.C. Supervision: S.O.-A., J.M.-L., J.F.A., L.F.-V. All authors critically reviewed the paper and approved the final version to be published.

\section{Data Availability Statement}

All the obtained data used to support the findings of this study are available from the corresponding author upon request.

\section{References}

1 Núñez-Álvarez C, Osborne NN. Blue light exacerbates and red light counteracts negative insults to retinal ganglion cells in situ and R28 cells in vitro. Neurochem Int. 2019 May; 125: 187-96.

2 Jaadane I, Villalpando Rodriguez GE, Boulenguez P, Chahory S, Carré S, Savoldelli M, et al. Effects of white light-emitting diode (LED) exposure on retinal pigment epithelium in vivo. J Cell Mol Med. 2017;21(12):3453-66.

3 Shang YM, Wang GS, Sliney D, Yang CH, Lee LL. White light-emitting diodes (LEDs) at domestic lighting levels and retinal injury in a rat model. Environ Health Perspect. 2014;122(3): 269-76.

4 Behar-Cohen F, Martinsons C, Viénot F, Zissis G, Barlier-Salsi A, Cesarini JPP, et al. Light-emitting diodes (LED) for domestic lighting: any risks for the eye? Prog Retin Eye Res. 2011 Jul;30(4):239-57.

5 Margrain TH, Boulton M, Marshall J, Sliney DH. Do blue light filters confer protection against age-related macular degeneration? Prog Retin Eye Res. 2004 Sep;23(5):523-31.

6 Krigel A, Berdugo M, Picard E, Levy-Boukris $\mathrm{R}$, Jaadane I, Jonet L, et al. Light-induced retinal damage using different light sources, protocols and rat strains reveals LED phototoxicity. Neuroscience. 2016 Dec;339:296-307.

7 Cheng QE, Gao J, Kim BJ, Ying GS. Design characteristics of geographic atrophy treatment trials: systematic review of registered trials in ClinicalTrials.gov. Ophthalmol Retina. 2018 Jun;2(6):518-25.

8 Jaadane I, Boulenguez P, Chahory S, Carré S, Savoldelli M, Jonet L, et al. Retinal damage induced by commercial light emitting diodes (LEDs). Free Radic Biol Med. 2015 Jul;84 373-84.
9 del Olmo-Aguado S, Núñez-Álvarez C, Osborne NN. Blue light action on mitochondria leads to cell death by necroptosis. Neurochem Res. 2016 Sep;41(9):2324-35.

10 Tao JX, Zhou WC, Zhu XG. Mitochondria as potential targets and initiators of the blue light hazard to the retina. Oxid Med Cell Longev. 2019;2019:6435364.

11 Cheng KC, Hsu YT, Liu W, Huang HL, Chen LY, He CX, et al. The role of oxidative stress and autophagy in blue-light-induced damage to the retinal pigment epithelium in zebrafish in vitro and in vivo. Int J Mol Sci. 2021 Feb; 22(3):1-17.

12 Chidlow G, Wood JP, Casson RJ. Pharmacological neuroprotection for glaucoma. Drugs. 2007;67(5):725-59.

13 Tezel G, Yang X, Luo C, Peng Y, Sun SL, Sun D. Mechanisms of immune system activation in glaucoma: oxidative stress-stimulated antigen presentation by the retina and optic nerve head glia. Invest Ophthalmol Vis Sci. 2007 Feb;48(2):705.

14 Chidlow G, Wood JPM, Casson RJ. Investigations into hypoxia and oxidative stress at the optic nerve head in a rat model of glaucoma. Front Neurosci. 2017 Aug; 11:478.

15 Del Olmo-Aguado S, Manso AG, Osborne NN. Light might directly affect retinal ganglion cell mitochondria to potentially influence function. Photochem Photobiol. 2012 Nov; 88(6):1346-55.

16 Osborne NN, Núñez-Álvarez C, Del OlmoAguado S. The effect of visual blue light on mitochondrial function associated with retinal ganglions cells. Exp Eye Res. 2014;128:814.

17 Boettner EA, Wolter JR. Transmission of the ocular media. Invest Ophthalmol Vis Sci. 1962;1(6):776-83.
18 Kessel L, Lundeman JH, Herbst K, Andersen TV, Larsen M. Age-related changes in the transmission properties of the human lens and their relevance to circadian entrainment. J Cataract Refract Surg. 2010 Feb;36(2):30812.

19 Sakanishi Y, Awano M, Mizota A, Tanaka M, Murakami A, Ohnuma K. Age-related changes in spectral transmittance of the human crystalline lens in situ. Ophthalmologica. 2012 Sep;228(3):174-80.

20 Werner JS, Spillmann L. UV-absorbing intraocular lenses: safety, efficacy, and consequences for the cataract patient. Graefes Arch Clin Exp Ophthalmol. 1989 May;227(3):248 56.

21 Henderson BA, Grimes KJ. Blue-blocking IOLs: a complete review of the literature. Surv Ophthalmol. 2010 May;55(3):284-9.

22 Downes SM. Ultraviolet or blue-filtering intraocular lenses: what is the evidence? Eye. 2016;30(2):215

23 Artigas JM, Felipe A, Navea A, Fandiño A, Artigas C. Spectral transmission of the human crystalline lens in adult and elderly persons: color and total transmission of visible light. Invest Ophthalmol Vis Sci. 2012 Jun;53(7): 4076-84.

24 Pipis A, Touliou E, Pillunat LE, Augustin AJ. Effect of the blue filter intraocular lens on the progression of geographic atrophy. Eur J Ophthalmol. 2015;25(2):128-33.

25 Mendoza-Mendieta ME, Lorenzo-Mejía AA Associated depression in pseudophakic patients with intraocular lens with and without chromophore. Clin Ophthalmol. 2016 Mar; 10:577-81. 
26 Mainster MA, Turner PL. Blue-blocking IOLs vs. short-wavelength visible light: hypothesisbased vs. evidence-based medical practice. Ophthalmology. 2011 Jan;118(1):1-2.

27 Artigas JM, Felipe A, Navea A, Artigas C, García-Domene MC. Spectral transmittance of intraocular lenses under natural and artificial illumination: criteria analysis for choosing a suitable filter. Ophthalmology. 2011 Jan; 118(1):3-8

28 Godley BF, Shamsi FA, Liang FQ, Jarrett SG, Davies S, Boulton M. Blue light induces mitochondrial DNA damage and free radical production in epithelial cells. J Biol Chem. 2005 Jun;280(22):21061-6.

29 Lascaratos G, Ji D, Wood JP, Osborne NN. Visible light affects mitochondrial function and induces neuronal death in retinal cell cultures. Vision Res. 2007 Apr;47(9):1191-201.

30 González-Menéndez I, Contreras F, GarcíaFernández JM, Cernuda-Cernuda R. Perinatal development of melanopsin expression in the mouse retina. Brain Res. 2011 Oct;1419: 12-8.

31 Cui Q, Ren C, Sollars PJ, Pickard GE, So KF. The injury resistant ability of melanopsin-expressing intrinsically photosensitive retinal ganglion cells. Neuroscience. 2015 Jan;284: 845-53.

32 Lazzerini Ospri L, Prusky G, Hattar S. Mood, the circadian system, and melanopsin retinal ganglion cells. Annu Rev Neurosci. 2017 Jul; 40:539-56.

33 Berson DM, Dunn FA, Takao M. Phototransduction by retinal ganglion cells that set the circadian clock. Science. 2002 Feb;295(5557): 1070-3.
34 Hankins MW, Peirson SN, Foster RG. Melanopsin: an exciting photopigment. Trends Neurosci. 2008 Jan;31(1):27-36.

35 Lucas RJ, Douglas RH, Foster RG. Characterization of an ocular photopigment capable of driving pupillary constriction in mice. Nat Neurosci. 2001;4(6):621-6.

36 King A, Gottlieb E, Brooks DG, Murphy MP, Dunaief JL. Mitochondria-derived reactive oxygen species mediate blue light-induced death of retinal pigment epithelial cells. Photochem Photobiol. 2004 May;79(5):470-5.

37 Chrysostomou V, Rezania F, Trounce IA, Crowston JG. Oxidative stress and mitochondrial dysfunction in glaucoma. Curr Opin Pharmacol. 2013 Feb;13(1):12-5.

38 Núñez-Álvarez C, Suárez-Barrio C, del Olmo Aguado S, Osborne NN. Blue light negatively affects the survival of ARPE19 cells through an action on their mitochondria and blunted by red light. Acta Ophthalmol. 2019 Feb; 97(1):e103-15.

39 Chen WJ, Wu C, Xu Z, Kuse Y, Hara H, Duh EJ. Nrf2 protects photoreceptor cells from photo-oxidative stress induced by blue light. Exp Eye Res. 2017 Jan;154:151-8.

40 Alaimo A, Liñares GG, Bujjamer JM, Gorojod RM, Alcon SP, Martínez JH, et al. Toxicity of blue led light and A2E is associated to mitochondrial dynamics impairment in ARPE-19 cells: implications for age-related macular degeneration. Arch Toxicol. 2019 May;93(5): 1401-15.
41 del Olmo-Aguado S, Núñez-Álvarez C, Osborne NN. Red light of the visual spectrum attenuates cell death in culture and retinal ganglion cell death in situ. Acta Ophthalmol. 2016 Sep;94(6):e481-91.

42 Lever JM, Boddu R, George JF, Agarwal A. Heme oxygenase- 1 in kidney health and disease. Antioxid Redox Signal. 2016 Jul;25(3): 165-83.

43 Kumar A, Takada Y, Boriek AM, Aggarwal $\mathrm{BB}$. Nuclear factor- $\kappa \mathrm{B}$ : its role in health and disease. J Mol Med. 2004 Jul;82(7):434-48.

44 Perkins ND. Post-translational modifications regulating the activity and function of the nuclear factor kappa B pathway. Oncogene. 2006 Oct;25(51):6717-30.

45 Tuna $\mathrm{D}$, Došlić $\mathrm{N}$, Mališ $\mathrm{M}$, Sobolewski AL, Domcke W. Mechanisms of photostability in kynurenines: a joint electronic-structure and dynamics study. J Phys Chem B. 2015 Feb; 119(6):2112-24

46 Zhu XF, Zou HD, Yu YF, Sun Q, Zhao NQ. Comparison of blue light-filtering IOLs and UV light-filtering IOLs for cataract surgery: a meta-analysis. PLoS One. 2012 Mar;7(3): e33013.

47 Downie LE, Wormald R, Evans J, Virgili G, Keller PR, Lawrenson JG, et al. Analysis of a systematic review about blue light-filtering intraocular lenses for retinal protection: understanding the limitations of the evidence. JAMA Ophthalmol. 2019 Jun;137(6):694-7.

48 Achiron A, Elbaz U, Hecht I, Spierer O, Einan-Lifshitz A, Karesvuo P, et al. The effect of blue-light filtering intraocular lenses on the development and progression of neovascular age-related macular degeneration. Ophthalmology. 2020 Jul;128(3):410-16. 